\title{
Spaltung des racemischen Histidins in seine optisch aktiven Komponenten.
}

\author{
Von \\ Emil Abderhalden und Arthur Weil.
}

(Aus dem physiologischen Institut der Universität Halle a. S.)

(Der Redaktion zugegangen am 7. März 1912.)

Von den meisten Aminosäuren sind neben der in der Natur vorkommenden Form der Racemkörper und der optische Antipode bekannt. Beim Histidin ist dies nicht der Fall. Die in der Natur vorkommende Form ist das l-Histidin. Wir haben dieses durch Erhitzen unter Druck mit Baryt racemisiert und dann das dl-Histidin mit Hilfe von d-Weinsäure in seine optisch aktiven Komponenten, das l-und d-Histidin, zerlegt. ${ }^{1}$ ) Dieser Weg erwies sich als außerordentlich brauchbar, und wir vermuten, daß sich racemisches Lysin und Arginin über das weinsaure Salz ebenfalls in die optisch aktiven Komponenten zerlegen lassen. Mit Versuchen nach dieser Richtung sind wir beschäftigt. Ferner konnten wir feststellen, daß auch die Monoaminosäuren, wie z. B. Leucin und Valin, sehr schön krystallisierende Salze nach Zugabe von d-Weinsäure geben. Ob es gelingen wird, Bedingungen ausfindig zu machen, um racemische Monoaminosäuren und vielleicht auch racemische Polypeptide mit Hilfe der d-Weinsäure in die optisch aktiven Bestandteile zu zerlegen, muß die weitere Erfahrung ergeben. Wir haben ferner dl-Histidin durch Hefezellen zu spalten versucht, und es endlich an Kaninchen verfüttert und aus dem Harn reines d-Histidin gewonnen. Bei dieser Gelegenheit haben wir die Eigenschaften von $\mathrm{dl}-, \mathrm{d}$ - und l-Histidin und einige ihrer Derivate studiert.

\section{Experimenteller Teil.}

\section{Darstellung von dl-Histidin aus l-Histidin.}

Als Ausgangsmaterial diente l-Histidin, das nach dem üblichen Verfahren aus Pferdeblut gewonnen worden war. Die vom

1) Anmerkung bei Korrektur. Vgl. den Schluß dieser Arbeit. Frank Lee Pyman hat diesen Weg 1911 schon eingeschlagen. Seine Mitteilung war uns entgangen. 
Serum getrennten roten Blutkörperchen blieben vier bis sechs Tage mit dem gleichen Volumen konzentrierter Salzsäure gemischt stehen und wurden dann sechs Stunden am Rückflußkühler gekocht. Die weitere Verarbeitung geschah nach den vorhandenen Vorschriften. ${ }^{1}$ ) Es empfiehlt sich, das Hydrolysenfiltrat mit Natronlauge bis zur amphoteren Reaktion zu neutralisieren und nicht, wie angegeben, bis zur schwach alkalischen. Man kann dann von dem ausfallenden Niederschlage leicht abfiltrieren und erhält ein hell- bis dunkelgelbes Filtrat, das nunmehr mit Sodalösung deutlich alkalisch gegen Lackmus gemacht wird. Das zeitraubende Kochen bis zur vollständigen Entfernung des Ammoniaks unterließen wir ebenfalls. ${ }^{1}$ ) Blieb der erwartete Niederschlag nach Zusatz der alkoholischen Sublimatlösung $(1 \mathrm{~kg}$ auf 10 l Blut) aus, so wurde weiter Sodalösung bis zur Bildung von groben Flocken zugefügt. Wir erhielten stets zunächst das Histidinmonochlorhydrat und in den letzten Mutterlaugen erst das Dichlorhydrat.

$\mathrm{Da}$ es sich bei unseren späteren Versuchen als bequemer erwies, wenn wir mit der freien Aminosäure arbeiteten, suchten wir nach neuen Verfahren, um diese aus dem Chlorhydrat zu isolieren. Die von Kossel angegebene Isolierung mit Silbersulfat $^{2}$ ) und das Fränkelsche Verfahren mit frisch gefälltem Silbercarbonat ${ }^{3}$ ) waren für größere Mengen zu kostspielig und zu umständlich. Wir versuchten zunächst durch Kochen mit Bleioxyd Histidin aus dem Chlorhydrat zu gewinnen: $130 \mathrm{~g}$, durch öfteres Umkrystallisieren aus kochendem Wasser gereinigtes Histidinmonochlorhydrat, wurden zwei Stunden lang, in ca. 1,5 l Wasser gelöst, mit einem Überschuß an Bleioxyd gekocht. Nach dem Erkalten wurde abfiltriert, der Rückstand ausgewaschen, bis das Filtrat mit Bromwasser keine Rotfärbung

1) Emil Abderhalden und Hans Einbeck, Studien über den Abbau des Histidins im Organismus des Hundes, Diese Zeitschrift, Bd. 62, Heft 4, S. 329, 1909.

2) A. Kossel, Über die basischen Stoffe des Zellkerns, Diese Zeitschrift, Bd. 22, S. 182, 1896.

s) S. Fraenkel, Über Darstellung und Konstitution des Histidins, Monatshefte für Chemie, Bd. 24, S. 230, 1903. 
mehr zeigte, und dann das Blei aus dem Filtrat mit Schwefel wasserstoff gefällt. Das im Vakuum bis zur Krystallisation eingeengte Filtrat gab nach mehrtägigem Stehen eine Ausbeute von $90 \mathrm{~g}$ Rohhistidin $=94 \%$ der Theorie.

Als wir das durch zweimaliges Umkrystallisieren aus etwa der zehnfachen Menge kochenden Wassers gereinigte Histidin auf sein Drehungsvermögen untersuchten, zeigte es sich, daß es stark racemisiert war. Das Ausgangsmaterial zeigte in den von Abderhalden und Einbeck ${ }^{1}$ ) angegebenen Konzentrationen eine spezifische Drehung von $+1,18^{\circ}$. $-0,4438 \mathrm{~g}$ Histidinmonochlorhydrat in 5,4544 $\mathrm{g}$ Wasser gelöst, $d=1,0295$, drehten bei filtriertem Gasglühlicht $+0,09^{\circ}\left( \pm 0,01^{\circ}\right)$ im $1 \mathrm{dm}-$ Rohr.

Das daraus mit Silbercarbonat isolierte Histidin ergab $[\alpha]_{\mathrm{D}}^{20^{\circ}}=-\mathbf{3 5 , 5 6}^{\circ} .0,3054 \mathrm{~g}$ Substanz in 9,9167 $\mathrm{g}$ Lösung, $\mathrm{d}=1,0143$, drehten die Ebene des polarisierten Lichtes $1,08^{\circ}$ $\left( \pm 0,02^{\circ}\right)$ nach links im $1 \mathrm{dm}-$ Rohr.

Das durch Kochen mit Bleioxyd isolierte Histidin besaß dagegen nur eine spezifische Drehung von - $\mathbf{2 4 , 5 8 ^ { 0 }}$.

$0,2144 \mathrm{~g}$ Substanz in 10,8121 $\mathrm{g}$ wässeriger Lösung, $\mathrm{d}=1,0054$, drehten $-0,49^{\circ}\left( \pm 0,02^{\circ}\right)$ im $1 \mathrm{dm}$-Rohr.

Zur Darstellung von optisch aktivem Histidin empfiehlt sich also das genannte Verfahren nicht. Dagegen erhielten wir sehr gute Resultate bei Anwendung von Lithiumhydroxyd: $\mathrm{Zu}$ einer abgewogenen Menge Histidinmonochlorhydrat, in wenig kaltem Wasser gelöst, wird die berechnete Menge NormalLithiumhydroxydlösung hinzugefügt, im Vakuum bei ca. $50^{\circ}$ bis zur Trockne eingedampft, mit wenig absolutem Alkohol extrahiert und der Rückstand aus etwa der zehnfachen Menge Wasser umkrystallisiert. Im Eisschrank schieden sich schnell glänzende Krystallblättchen ab. Die Ausbeute beträgt ca. 90\% der Theorie. 0,2928 $\mathrm{g}$ Substanz in 8,3620 $\mathrm{g}$ wässeriger Lösung, $\mathrm{d}=1,0136$, drehten im $1 \mathrm{dm}-\mathrm{Rohr}$ bei Natriumlicht $-1,40^{\circ}$.

$$
[\alpha]_{\mathrm{D}}^{20^{\circ}}=-39,44^{\circ} \text {. }
$$

F.: Bei raschem Erhitzen Braunfärbung bei etwa $255^{\circ}$

1) Siehe oben S. 332 . 
(nicht korr.). Zersetzung unter Aufschäumen bei $279^{\circ}$ (nicht korr. $=285^{\circ}$ korr.).

Fränkel ${ }^{1}$ ) gibt F. mit $253^{\circ}$ an, unter Aufschäumen und Zersetzung.

0,1784 g Substanz verbrauchten nach Kjeldahl $34,3 \mathrm{ccm}$ $\mathrm{n}_{1 / 10}-\mathrm{H}_{2} \mathrm{SO}_{4}$.

Berechnet für $\mathrm{C}_{6} \mathrm{H}_{9} \mathrm{~N}_{3} \mathrm{O}_{2}(155,1)$ : Gefunden :

$$
\mathrm{N}=27,10 \% \quad 26,94 \% \text {. }
$$

Der einfachste und bequemste Weg zur Isolierung der freien Base aus dem Chlorhydrat ist folgender: In eine möglichst konzentrierte wässerige Lösung von Histidinmonochlorhydrat wird bis zur schwachen Übersättigung Ammoniakgas eingeleitet. Beim Einengen auf dem Wasserbade scheiden sich schnell große Krystalle $a b$, die aus Wasser umkrystallisiert werden.

F.: Bei $279^{\circ}$ (unkorr.) Zersetzung unter Aufschäumen. Es tritt keine Racemisierung durch Ammoniak im Überschuß ein.

0,2161 g Substanz in 9,8168 g wässeriger Lösung, $\mathrm{d}=1,0082$, drehen im $1 / 2 \mathrm{dm}$-Rohr bei filtriertem Gasglühlicht, $-0,44^{0}\left( \pm 0,01^{0}\right)$.

$$
[\alpha]_{\mathrm{D}}^{200}=-\mathbf{3 9 , 6 5}{ }^{\circ} \text {. }
$$

Um aus optisch aktivem Histidin den Racemkörper darzustellen, erhitzte Fränkel Histidin mit 20\% iger Salzsäure im Schießrohr auf $160^{\circ 2}$ ).

Bedeutend leichter wird die Racemisierung durch Barytwasser bewirkt. $1 \mathrm{~g}$ Histidinmonochlorhydrat $+100 \mathrm{ccm}$ konzentrierte Salzsäure $(d=1,19)$ war nach 30 stündigem Kochen am Rückflußkühler gar nicht racemisiert. Dagegen zeigten $2 \mathrm{~g}$ Histidinmonochlorhydrat $+20 \mathrm{~g}$ Baryt $+600 \mathrm{ccm}$ Wasser nach 20 stündigem Kochen am Rückflußkühler ein Sinken der ursprünglichen Drehung von $-0,16^{\circ}$ im $1 \mathrm{dm}$-Rohr auf $-0,05^{\circ}$.

Eine vollständige Racemisierung erreichten wir schneller durch Erhitzen im Autoklaven. Die folgenden Zahlen für die Versuchsdauer und den Atmosphärendruck sind die durch ver-

1) 1. c. S. 232 .

2) Sigmund Fränkel, Abbau des Histidins. Beiträge z. chemischen Physiologie und Pathologie, Bd. 8, S. 160, 1906. 
schiedene Versuche ermittelten günstigsten Werte. Bei 7-8 Atmosphären Druck tritt leicht Zersetzung unter Schwarzfärbung ein. Bei kürzerer Versuchsdauer wird keine vollkommene Racemisierung erreicht.

$50 \mathrm{~g}$ Histidinmonochlorhydrat in $300 \mathrm{ccm}$ heißen Wassers gelöst werden mit $150 \mathrm{~g}$ Baryt 5 Stunden lang bei 5 Atmosphären Druck im Autoklaven erhitzt. Nach dem Öffnen macht sich starker Ammoniakgeruch bemerkbar und rotes Lackmuspapier wird durch die Dämpfe tiefblau gefärbt. Das hellbraun gefärbte Gemisch wird in 51 kochenden Wassers gelöst und heiß abfiltriert. Der Rückstand wiegt, bei $80^{\circ}$ getrocknet, $26 \mathrm{~g}$ und erwies sich als Baryumcarbonat. Aus dem Filtrat wird der Baryt quantitativ mit Schwefelsäure gefällt und der Niederschlag zweimal mit je $500 \mathrm{ccm}$ Wasser ausgekocht. Die vereinigten Filtrate werden im Vakuum bis auf etwa 1 l eingeengt und zweimal eine Stunde lang mit je 80 g Bleioxyd gekocht. Nach dem Erkalten wird filtriert und nach dem Auswaschen der Rückstände das Blei mit Schwefelwasserstoff gefällt. Nach dem Entfernen des letzteren aus dem Filtrat durch Durchleiten von Luft wird mit Tierkohle entfärbt und schließlich im Vakuum bei etwa $50^{\circ}$ bis zur beginnenden Krystallisation eingeengt. Die vereinigten Krystallfraktionen gaben nach einmaligem Umkrystallisieren aus etwa der 10 fachen Menge kochenden Wassers eine Ausbeute von $19 \mathrm{~g}$ dl-Histidin $=51 \%$ der Theorie.

Zur Analyse wurde die Substanz nochmals aus der 20 fachen Menge kochenden Wassers umkrystallisiert.

$0,1614 \mathrm{~g}$ gaben $0,2735 \mathrm{~g} \mathrm{CO}_{2}$ und $0,0771 \mathrm{~g} \mathrm{H}_{2} \mathrm{O}$.

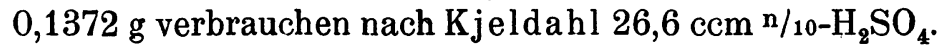

Berechnet für $\mathrm{C}_{6} \mathrm{H}_{9} \mathrm{~N}_{3} \mathrm{O}_{2}(155,1)$ :

Gefunden:

$$
\begin{array}{lr}
\text { C } 46,42 \% & \text { C } 46,22 \% \\
\text { H } 5,85 \% & \text { H } 5,38 \% \\
\text { N } 27,10 \% & \text { N } 27,16 \%
\end{array}
$$

In 3\% iger Lösung zeigt die Substanz im $1 \mathrm{dm}-$ Rohr keine Drehung des polarisierten Lichtes.

F. : Bei raschem Erhitzen Braunfärbung zwischen 255 und $260^{\circ}$. Zersetzung unter Aufschäumen gegen 279-280 
( $=285-286^{\circ}$ korr.). dl-Histidin besitzt entgegen dem fade schmeckenden l-Histidin einen schwach süßen Geschmack.

Es ist in kaltem Wasser schwer löslich, in kochendem etwa 1 : 20 ; unlöslich in Alkohol, Äther, Aceton, Chloroform. Aus heißem Wasser krystallisiert es in viereckigen Prismen mit quadratischer Grundfläche und einem Achsenverhältnis von etwa $1: 1: 3$.

Mit Bromwasser erhält man beim Erwärmen Rotviolettfärbung, die in Schwarz übergeht.

Eigenschaften des dl-Histidindichlorhydrats: dlHistidin in etwa der 10 fachen Menge warmer, konzentrierter Salzsäure gelöst, scheidet sich beim langsamen Verdunsten im Exsikkator in großen Krystallen als Dichlorhydrat ab. Abgesaugt und im Exsikkator über Schwefelsäure getrocknet, zeigte die Substanz beim raschen Erhitzen im Kapillarrohr gegen $237^{\circ}$ (unkorr.) Erweichen, und ohne zu schmelzen gegen 250-255 (unkorr.). Zersetzung unter Aufschäumen. Geschmack stark sauer.

Fränkel gibt für das racemisierte Histidindichlorhydrat einen Schmelzpunkt von $220^{\circ}$ C. an. ${ }^{1}$ )

\section{Analysen:}

$0,1806 \mathrm{~g}$ Substanz verbrauchten $15,7 \mathrm{ccm} \mathrm{n} / 10 \quad \mathrm{AgNO}_{3}$.

$0,1154 \mathrm{~g}$ Substanz verbrauchten nach $\mathrm{Kjeld}$ ahl $28,15 \mathrm{ccm}$ $\mathrm{n} / 10-\mathrm{H}_{2} \mathrm{SO}_{4}$. Für $\mathrm{C}_{6} \mathrm{H}_{9} \mathrm{~N}_{3} \mathrm{O}_{2} \cdot 2 \mathrm{HCl}(228,04)$.

Berechnet:

Cl $31,10 \%$ N $18,43 \%$
Gefunden:

Cl $30,83 \%$ N $18,31 \%$.

II. Spaltung von dl-Histidin in seine optisch aktiven Komponenten.

Zur Spaltung von racemischen Aminosäuren stehen uns in der Hauptsache bis jetzt zwei Methoden zur Verfügung. Bei der einen, von Emil Fischer ausgearbeiteten Methode - Darstellung der Benzoyl- resp. Formylverbindung und Trennung der beiden mit Hilfe des Brucinsalzes - erhalten wir beide optisch aktiven Komponenten. Die zweite Methode, die sogenannte biologische, liefert in mehr oder weniger guter Ausbeute die in der Natur nicht vorkommende optisch aktive Komponente,

i) a. a. O., S. 160 . 
während die in der Natur vorkommende vom angewandten Organismus verwendet wird. Entweder benutzt man Mikroorganismen, z. B., nach dem Vorschlage von Felix Ehrlich Hefe, zur Spaltung der racemischen Verbindung, oder man verfüttert den Racemkörper an ein höher organisiertes Tier (W ohl gemuth). Wir haben zunächst versucht, Formyl-dl-histidin mit Hilfe von Brucin zu spalten. Es gelang uns nicht, optisch aktives Histidin auf diesem Wege zu erhalten.

1. Versuch, Formyl-dl-histidin mittels des Brucinsalzes zu spalten.

a) Darstellung von Formyl-1-histidin.

Das F ormyl-l-histidin ist bereits von Emil Fischer und Lee H. Cone dargestellt worden. ${ }^{1}$ ) Doch sind in der betreffenden Arbeit keine Angaben über das spezifische Drehungsvermögen gemacht. Wir stellten deshalb nach ihren Vorschriften aus $5 \mathrm{~g}$ l-Histidin $-[\alpha]_{\mathrm{D}}^{200}=-39,44-$ die Formylverbindung dar. Beim Ausfällen des durch Extraktion mit heißem Amylalkohol gewonnenen Rohproduktes aus der wässerigen Lösung mit Alkohol erhielten wir eine I. Fraktion von $1,8 \mathrm{~g}$, die durch zweimaliges Umkrystallisieren aus verdünntem Alkohol gereinigt und im Exsikkator über Schwefelsäure getrocknet wurde. F.: Zersetzung unter Aufschäumen bei $198^{\circ}$ (korr. $202^{\circ}$ ).

$0,2019 \mathrm{~g}$ Substanz in 9,3086 $\mathrm{g}$ wässeriger Lösung, $\mathrm{d}=$ 1,0077 , drehen im $1 \mathrm{dm}-\mathrm{Rohr}$ bei Natriumlicht $+1,24^{\circ}\left( \pm 0,02^{\circ}\right)$.

$$
[\alpha]_{\mathrm{D}}^{20^{\circ}}=+56,73^{\circ}\left( \pm 0,91^{\circ}\right) \text {. }
$$

b) Darstellung von Formyl-dl-histidin.

17,5 g dl-Histidin werden mit $29 \mathrm{~g}$ wasserfreier Ameisensäure 3 Stunden auf dem Wasserbade erhitzt. Die Lösung wird im Vakuum bis zum Sirup eingeengt. Wieder wird 3 Stunden lang mit $29 \mathrm{~g}$ Ameisensäure erhitzt, eingeengt und die Formylierung noch ein drittes Mal wiederholt. Der so gewonnene braune Sirup

1) Emil Fischer und Lee H. Cone, Synthese von Polypeptiden. XXVII. Derivate des Histidins. Annalen der Chemie und Pharmacie, Bd. 363, S. 107, 1908.

Hoppe-Seyler's Zeitschrift f. physiol. Chemie. LXXVII. 
wird 14 Tage lang im Vakuumexsikkator über Schwefelsäure und Ätznatron getrocknet, dann in etwa $15 \mathrm{ccm}$ warmen Wassers gelöst und mit $100 \mathrm{ccm}$ Alkohol $+20 \mathrm{ccm}$ Äther versetzt. Im Eisschrank fällt ein flockiger, weißer Niederschlag aus, der nach dem Absaugen nochmals in wenig Wasser gelöst und mit Alkohol und Äther gefällt und schließlich durch Umkrystallisieren aus etwa $50 \%$ igem Alkohol gereinigt wird.

Ausbeute: $14,5 \mathrm{~g}=70 \%$ der Theorie.

$0,1976 \mathrm{~g}$ Substanz geben $0,3324 \mathrm{~g} \mathrm{CO}_{2}$ und $0,0824 \mathrm{~g} \mathrm{H}_{2} \mathrm{O}$.

$0,1559 \mathrm{~g}$ verbrauchten nach K jel dahl $25,4 \mathrm{ccm}{ }^{\mathrm{n}} / 10-\mathrm{H}_{2} \mathrm{SO}_{4}$. Berechnet für $\mathrm{C}_{7} \mathrm{H}_{9} \mathrm{~N}_{3} \mathrm{O}_{3}$ : C. $45,88 \% ; \mathrm{H} \mathrm{4,96 \%} ; \mathrm{N} 22,96 \%$. Gefunden:

C $45,87 \%$; H $4,67 \%$; N $22,82 \%$.

F.: Sintert bei $195^{\circ}$; Aufschäumen bei $203-204^{\circ}=207$ bis $208^{\circ}$ korr.). Formyl-dl-histidin löst sich in kaltem Wasser wenig, leicht in warmem (etwa 1:1); es ist schwer löslich in absolutem Alkohol und in Pyridin, leichter in 50\% igem Alkohol, fast unlöslich in Äther, Aceton und Chloroform. Aus verdünntem Alkohol krystallisiert es in Doppelpyramiden (Oktaëdern). Geschmack : bitter-sauer.

c) Darstellung des Brucinsalzes des Formyl-dl-histidins.

$10 \mathrm{~g}$ Formyl-dl-histidin werden in $1500 \mathrm{ccm}$ heißen absoluten Alkohols gelöst und $21,5 \mathrm{~g}$ Brucin in absolutem Alkohol gelöst hinzugefügt. Da nach mehrtägigem Stehen im Eisschrank keine Krystallisation erfolgt, wird allmählich im Vakuum bei $40^{\circ}$ solange eingeengt, bis schließlich bei $100 \mathrm{ccm}$ in der Kälte die Krystallisation beginnt. Nach 8 Tagen haben sich 15,1 g Krystalle abgeschieden, die abgesaugt und mit Alkohol gewaschen wurden. Die Mutterlauge wurde im Vakuum zur Trockne verdampft und aus beiden Teilen nach den Vorschriften von Emil Fischer und 0 . Warburgi ${ }^{1}$ ) das Brucin durch $\mathrm{Na}$ tronlauge und der Formylrest durch Hydrolyse mit Salzsäure abgespalten. Aus der salzsauren Lösung wurde das Histidin durch Sublimat gefällt, der Niederschlag, wie oben angegeben,

1) Emil Fischer und Otto Warburg, Spaltung des Leucins in die optisch aktiven Komponenten, Ber. d. Deutsch. chem. Ges., Jg. 38, S. 3997, 1905. 
verarbeitet und schließlich aus dem Chlorhydrat Histidin durch Lithiumhydroxyd gewonnen. Es erwies sich bei beiden Trennungen als optisch inaktiv.

Ein zweiter Versuch führte zu dem gleichen Ergebnis: $14 \mathrm{~g}$ Formyl-dl-histidin + 30,1 g Brucin in $1500 \mathrm{ccm}$ absoluten Alkohols gelöst und nach dem Abkühlen im Vakuum bei $40^{\circ}$ auf $100 \mathrm{ccm}$ eingeengt. Da keine Krystallisation erfolgt, wird nach 2 Tagen auf $50 \mathrm{ccm}$ eingeengt. Die nach 8 Tagen ausgefallenen Krystalle werden abgesaugt und, ebenso, wie das Filtrat, wie oben geschildert, verarbeitet. Das zurückgewonnene Histidin war optisch inaktiv. Es war somit eine Trennung der beiden Komponenten nicht geglückt.

Da sich bei verschiedenen Versuchen ergeben hatte, daß dl- und l-Formylhistidin sich in wässeriger Lösung beim Einengen im Vakuum schon bei $40^{\circ}$ zersetzen - das Destillat reagierte stark sauer und ließ deutlich Ameisensäuregeruch erkennen -, versuchten wir die zur Auflösung des Formylkörpers nötigen, großen Alkoholmengen und das nachherige Einengen auf folgende Weise zu vermeiden:

$5 \mathrm{~g}$ dl-Formylhistidin wurden in $7 \mathrm{ccm}$ heißen Wassers gelöst und zu einer heißen Lösung von $10,7 \mathrm{~g}$ Brucin in $50 \mathrm{ccm}$ absoluten Alkohols hinzugefügt. Dann wurde mit $10 \mathrm{~g}$ Magnesiumsulfat etwa 15 Minuten geschüttelt und heiß abgesaugt. Im Eisschrank fielen nach etwa 14 Tagen Krystalle aus, die abgesaugt und, wie oben angegeben, verarbeitet, wiederum inaktives Histidin gaben.

2. Biologische Spaltung des dl-Histidins.

Nach den von Felix Ehrlich gegebenen Vorschriften') versuchten wir zunächst durch Spaltung mit Hefe aus dem Racemkörper das d-Histidin zu gewinnen.

Versuch 1 : 7,5 g dl-Histidin wurden mit $300 \mathrm{~g}$ Rohrzucker in $1600 \mathrm{ccm}$ Leitungswasser gelöst und $150 \mathrm{~g}$ Hefe, Rasse M des Instituts für Gärungsgewerbe in Berlin, fein zerrieben hinzugefügt. Nach vier Tagen war die Kohlensäureentwicklung beendet. Das zeitraubende Absaugen durch Filterkerzen oder Puckahlfilter um-

1) Handbuch der biochemischen Arbeitsmethoden, herausgegeben von Emil Abderhalden, Bd. 2, S. 563, 1910. 
gingen wir, indem wir einfach durch eine dünne Schicht Tierkohle absaugten, was längstens eine Stunde dauerte. Den Filterrückstand kochten wir zweimal mit Wasser aus. Die klaren Filtrate wurden mit Sodalösung schwach alkalisch gemacht und mit $10 \mathrm{~g}$ Sublimat, in $40 \mathrm{ecm}$ heißen Alkohols gelöst, versetzt. Der ausfallende Niederschlag wurde durch mehrmaliges Dekantieren mit Wasser salzfrei gewaschen, mit Schwefelwasserstoff zersetzt und nach dessen Entfernung durch Schütteln mit frisch gefälltem Silbercarbonat vom Chlor befreit. Aus dem Filtrat wurde das überschüssige Silber mit Schwefelwasserstoff entfernt und das Filtrat vom Silbersulfid im Vakuum (nicht auf dem Wasserbade, da Histidinlösungen hierbei leicht unter Braunfärbung zersetzt werden) bei etwa $50^{\circ}$ zur Trockne verdampft. Es hinterblieb ein fester Rückstand, der im Exsikkator getrocknet, $3,2 \mathrm{~g}$ wog. Aus $30 \mathrm{ccm}$ kochenden Wassers umkrystallisiert, schied sich im Eisschrank eine I. Fraktion von $1,9 \mathrm{~g}$ ab, die nach zweimaligem Umkrystallisieren aus Wasser sich unter Aufschäumen bei $278^{\circ}$ zersetzte, mit Bromwasser die bekannte Histidinreaktion gab und optisch inaktiv war. $\mathrm{n} / 10-\mathrm{H}_{8} \mathrm{SO}_{4}$.

$0,1537 \mathrm{~g}$ Substanz verbrauchen nach Kjeldahl 29,6 cem

Berechnet für $\mathrm{C}_{6} \mathrm{H}_{9} \mathrm{~N}_{3} \mathrm{O}_{2}(155,1)$

Gefunden :

$27,10 \% \mathrm{~N}$

$27,0 \% \mathrm{~N}$.

Aus der Mutterlauge der I. Fraktion wurde eine II. Fraktion gewonnen, die roh $0,2 \mathrm{~g}$ wog, dieselben Eigenschaften, wie die erste, zeigte und ebenfalls optisch inaktiv war. Die Mutterlauge hiervon wurde durch Kochen mit Tierkohle entfärbt und unter Zusatz von wenig Alkohol vorsichtig eingeengt. Es wurde schließlich im Eisschranke eine III. Fraktion gewonnen, die roh $0,65 \mathrm{~g}$ wog und zur Analyse aus kochendem Wasser umkrystallisiert wurde.

$0,1774 \mathrm{~g}$ geben $0,3025 \mathrm{~g} \mathrm{CO}_{2}$ und $0,0936 \mathrm{~g} \mathrm{H}_{2} \mathrm{O}$.

$0,1109 \mathrm{~g}$ verbrauchen nach $\mathrm{Kjeldahl} 21,7 \mathrm{~cm} \mathrm{n} / 10-\mathrm{H}_{2} \mathrm{SO}_{4}$.

Für $\mathrm{C}_{6} \mathrm{H}_{9} \mathrm{~N}_{3} \mathrm{O}_{2}(155,1)$

Berechnet: C $46,42 \%$;

H $5,85 \%$;

N $27,10 \%$.

Gefunden: C $46,51 \%$;

H $5,90 \%$;

N 27,4 \%. 
$0,1523 \mathrm{~g}$ in 6,8756 $\mathrm{g}$ wässeriger Lösung, $d=1,006$, drehen im $1 \mathrm{dm}-\mathrm{Rohr}+0,23^{\circ}\left( \pm 0,01^{\circ}\right)$.

$$
[\alpha]_{\mathrm{D}}^{200}=+10,32^{\circ} .
$$

F.: Zersetzung unter Aufschäumen bei $272^{\circ}$ (unkorr.). Mit Bromwasser starke Histidinreaktion. Geschmack: süß.

Die ersten beiden Fraktionen wurden so als racemisches Histidin, die dritte als ein Gemisch von d-Histidin und Racemkörper identifiziert.

Weitere Versuche, unter anderen Mengenverhältnissen und mit anderen Heferassen ausgeführt, gaben auch keine besseren Resultate.

Versuch II. $1 \mathrm{~g}$ dl-Histidin mit $30 \mathrm{~g}$ Zucker, $250 \mathrm{ccm}$ Wasser und $15 \mathrm{~g}$ Hefe, Rasse 12, angesetzt. 3 Tage vergoren. Verarbeitung, wie oben.

Endprodukt 0,1 g. F.: Schwarzfärbung $256^{\circ}$, Zersetzung $278^{\circ}$ (korr.).

$0,0346 \mathrm{~g}$ Substanz in $8,0986 \mathrm{~g}$ wässeriger Lösung, $\mathrm{d}=$ 1,0035 , drehen im $1 \mathrm{dm}-\mathrm{Rohr}^{\circ}+0,07^{\circ}\left( \pm 0,01^{\circ}\right)$.

$$
[\alpha]_{\mathrm{D}}^{200}=+16,37^{\circ} \text {. }
$$

Versuch III. $2 \mathrm{~g}$ dl-Histidin $+50 \mathrm{~g}$ Zucker $+400 \mathrm{ccm}$ Leitungswasser $+25 \mathrm{~g}$ Hefe Rasse XII. Gärung 5 Tage. Ausbeute rein: 0,2 g. F.: $276^{\circ}$ (unkorr.).

$0,1980 \mathrm{~g}$ Substanz in 7,5328 $\mathrm{g}$ wässeriger Lösung, $\mathrm{d}=$ 1,007 , drehen $+0,06^{\circ}\left( \pm 0,01^{\circ}\right)$.

$$
[\alpha]_{\mathrm{D}}^{200}=+2,27^{0} .
$$

Versuch IV. $10 \mathrm{~g}$ dl-Histidin $+800 \mathrm{~g}$ Zucker $+2500 \mathrm{ccm}$ $\mathrm{H}_{2} \mathrm{O}+400 \mathrm{~g}$ Hefe Rasse M. 5 Tage.

Ausbeute $0,3 \mathrm{~g} . \quad[\alpha]_{\mathrm{D}}^{200}=+\mathbf{3 , 3 6}^{\circ}$.

$0,1982 \mathrm{~g}$ Substanz in 9,5541 $\mathrm{g}$ Lösung, $\mathrm{d}=1,0055$, drehen im dm-Rohr $+0,07^{\circ}\left( \pm 0,01^{\circ}\right)$.

Da es uns mit der Hefespaltung nicht gelang, genügende Mengen optisch reinen d-Histidins zu gewinnen, versuchten wir durch Verfütterung von dl-Histidin an Kaninchen zum Ziele zu gelangen. 
Ein Kaninchen von etwa $2 \mathrm{~kg}$ Gewicht erhielt nach zweitägigem Hungern $5 \mathrm{~g}$ dl-Histidin in $300 \mathrm{ccm}$ Wasser gelöst innerhalb $1^{1 / 2}$ Stunden in drei Portionen vermittelst der Schlundsonde. In der Vorperiode besaß der Harn schwach alkalische Reaktion. Mit Bleiacetatlösung (15 ccm auf $100 \mathrm{ccm}$ Harn) geklärt, zeigte er im $1 \mathrm{dm}-\mathrm{Rohr}$ eine Drehung der Polarisationsebene um $+0,07 \mathrm{~g}$. Reaktion mit Bromwasser negativ.

12 Stunden nach der Fütterung untersucht, erhielten wir dieselben Resultate. Harnmenge $340 \mathrm{ccm}$. 36 Stunden nach der Eingabe des dl-Histidins war die Reaktion gegen Lackmus stark alkalisch. Die Drehung im $1 \mathrm{dm}-$ Rohr betrug $-0,15^{\circ}$. Beim Erwärmen mit einigen Tropfen Bromwasser trat deutliche Rotfärbung auf, die beim Erkalten allmählich in Dunkelviolettrot überging. 48 Stunden nach der Fütterung war ebenfalls noch schwach positive Reaktion mit Bromwasser vorhanden. $\alpha$ im $1 \mathrm{dm}-$ Rohr $+0,03^{\circ}$. Die vereinigten Harnmengen des ersten bis dritten Tages nach der Fütterung wurden mit Sodalösung schwach alkalisch gemacht und das Histidin mit $50 \mathrm{~g}$ Sublimat, in $200 \mathrm{ccm}$ Alkohol gelöst, gefällt. Die weitere Verarbeitung der Niederschläge war die übliche. In der Lösung des Chlorhydrats wurde der Chlorgehalt titrimetrisch bestimmt und die berechnete Menge Normal-Lithiumhydroxydlösung hinzugefügt. Im Vakuum wurde zur Trockne verdampft und der Rückstand mit $50 \mathrm{ccm}$ absoIuten Alkohols extrahiert. Das so gewonnene Rohprodukt wog im Exsikkator getrocknet 2,0 g. Aus $20 \mathrm{ccm}$ Wasser unter Zusatz von Tierkohle umkrystallisiert, schied sich bald eine I. Fraktion in glänzenden Blättchen $\mathrm{ab}$, die durch Umkrystallisieren aus kochendem Wasser gereinigt $0,8 \mathrm{~g}$ wog.

$0,1174 \mathrm{~g}$ verbrauchen $22,65 \mathrm{ccm} n / 10-\mathrm{H}_{2} \mathrm{SO}_{4}$.

Für $\mathrm{C}_{6} \mathrm{H}_{9} \mathrm{~N}_{3} \mathrm{O}_{2}(155,10)$.

Berechnet:
Gefunden: $27,10 \%$.
N 27,03\%.

F.: Bei raschem Erhitzen Braun- bis Schwarzfärbung gegen $250^{\circ}$; Zersetzung unter Aufschäumen bei $281^{\circ}$ (unkorr . $=287^{\circ}$ korr.).

Die reine Substanz löst sich schwer in kaltem Wasser", 
in heißem etwa $1: 10$. Sie ist unlöslich in Alkohol, Äther, Chloroform, Aceton.

Das d-Histidin schmeckt im Gegensatz zum etwas bitter bis fade schmeckenden l-Histidin sü B, wie etwa Rohrzucker. Mit Bromwasser gibt es die bekannte Reaktion.

d-Histidin krystallisiert aus Wasser in langen schmalen Tafeln von der Gestalt eines Rechteckes, an das auf der einen Schmalseite ein gleichseitiges, auf der andern ein ungleichseitiges Dreieck angesetzt ist:

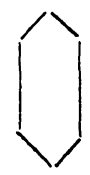

1-Histidin zeigt die gleiche Krystallform.

$0,2310 \mathrm{~g}$ Substanz in 10,2865 $\mathrm{g}$ wässeriger Lösung, $\mathrm{d}=1,0093$, drehen im $1 \mathrm{dm}$-Rohr bei Natriumlicht $+0,9^{\circ}$ $\left( \pm 0,02^{\circ}\right)$.

$$
[\alpha]_{\mathrm{D}}^{200}:+40,15^{\circ}\left( \pm 0,88^{\circ}\right) \text {. }
$$

Die Mutterlauge der ersten Fraktion wurde mit etwa dem 5 fachen Volumen absoluten Alkohols versetzt, der entstehende Niederschlag abfiltriert und aus kochendem Wasser mit Tierkohle umkrystallisiert.

Die II. Fraktion wurde zweimal aus Wasser umkrystallisiert. Die spezifische Drehung betrug nur $+\mathbf{1 9 , 6 1}^{\circ}$.

$0,1262 \mathrm{~g}$ in $7,3318 \mathrm{~g}$ wässeriger Lösung, $\mathrm{d}=1,0075$, drehten im ${ }^{1 / 2} \mathrm{dm}-$ Rohr bei Natriumlicht $+0,17^{\circ}\left( \pm 0,01^{\circ}\right)$.

F.: Bei $275^{\circ}$ (= $280^{\circ}$ korr.) Aufschäumen.

3. Spaltung von dl-Histidin mit Hilfe von d-Weinsäure.

Nachdem wir gefunden hatten, daß die Spaltung des racemischen Histidins in die optisch aktiven Komponenten mit Hilfe der Brucinverbindnngen der Formylkörper nicht möglich ist, suchten wir, abweichend von dem bis jetzt bei den Aminosäuren allgemein angewandten Verfahren, die Gewinnung des d-und l-Histidins aus dl-Histidin mittels einer optisch aktiven Säure auszuführen. 
Wir wählten als optisch aktive Säure d-Weinsäure, die auch von Flächer ${ }^{1}$ ) zur Spaltung von dl-Suprarenin in d- und l-Suprarenin verwendet worden ist.

Um die Eigenschaften des weinsauren Histidins kennen zu lernen, stellten wir zunächst das

\section{saure d-weinsaure l-Histidin}

dar. $1 \mathrm{~g}$ l-Histidin wird in $20 \mathrm{ccm}$ kochenden Wassers gelöst und $1 \mathrm{~g} \mathrm{~d}$-Weinsäure (etwas mehr als für $1 \mathrm{Mol}$. berechnet: $0,97 \mathrm{~g}$ ), in etwa $5 \mathrm{ccm}$ heißen Wassers gelöst, hinzugefügt. Beim Einengen im Exsikkator über Schwefelsäure bei Zimmertemperatur hinterbleibt ein zäher Sirup, der erst bei schärferem Trocknen im Vakuum krystallinisches Gefüge annimmt. Mit absolutem Alhohol verrieben, wird er in eine weiße, feinkrystallinische Substanz verwandelt, die sich gut aus etwa $30 \mathrm{ccm}$ verdünnten (ca. 80\%) Alkohols umkrystallisieren läßt.

Zur Analyse wird nochmals umkrystallisiert und im Exsikkator über Schwefelsäure getrocknet.

0,1549 g Substanz geben $0,2207 \mathrm{~g} \mathrm{CO}_{2}$ und $0,0700 \mathrm{~g} \mathrm{H}_{\mathbf{z}} \mathrm{O}$. $0,1462 \mathrm{~g}$ verbrauchen nach $\mathrm{Kjeldahl} 14,\left.3 \mathrm{ccm}{ }^{\mathrm{n}}\right|_{10}-\mathrm{H}_{2} \mathrm{SO}_{4}$.

Berechnet für Gefunden :

$$
\mathrm{C}_{6} \mathrm{H}_{3} \mathrm{~N}_{3} \mathrm{O}_{2} \cdot \mathrm{C}_{4} \mathrm{H}_{6} \mathrm{O}_{6}(305,16) \text { : }
$$
C: $39,33 \%$
C: $39,07 \%$
$\mathrm{H}: \quad 4.95 \%$
$\mathrm{H}: \quad 5,06 \%$
$\mathrm{N}: 13,77 \%$
$\mathrm{C}: 13,70 \%$

F.: Erweicht gegen $175^{\circ}$ (unkorr.) (korr. $178^{\circ}$ ). Zerset.zt sich unter Aufschäumen gegen $182^{\circ}$ (unkorr.). Bei langsamem Erhitzen Erweichung bei ca. $155^{\circ}$, F. $169-171^{\circ}$, Zersetzung unter Aufschäumen gegen $180^{\circ}$ (unkorr.). Das saure d-weinsaure l-Histidin löst sich leicht in kaltem und heißem Wasser, warmem verdünntem Alkohol (bis ca. 80\%); es ist fast unlöislich in absolutem Alkohol und Chloroform; unlöslich in Äther, Aceton, Methylalkohol.

Geschmack: schwach sauer mit fadem Nachgeschmacik.

1) Franz Flächer, Über die Spaltung des synthetischen dl-Siuprarenins in seine optisch aktiven Komponenten, Diese Zeitschrift, Bd. 5i8, Heft 3, S. 189, 1908. 
Gegen Lackmus reagiert es stark sauer und gibt mit Bromwasser die bekannte Histidinreaktion. Aus verdünntem Alkohol krystallisiert es in dreieckigen Blättchen, die mit der Basis zu morgensternartigen Drusen vereinigt sinł. 0,1588 $\mathrm{g}$ Substanz in $8,3834 \mathrm{~g}$ wässeriger Lösung, $\mathrm{d}=1,0085$, drehen im 1/2 dm-Rohr bei filtriertem Gasglühlicht $+0,15^{0}\left( \pm 0,01^{\circ}\right)$.

$$
[\alpha]_{\mathrm{D}}^{20^{\circ}}=+15,70^{\circ}\left( \pm 1,04^{\circ}\right) \text { für } \mathrm{c}=1,9104 \text { (s. unten). }
$$

Spaltung des racemischen Histidins.

$3,3 \mathrm{~g}$ dl-Histidin werden in $40 \mathrm{ccm}$ kochenden Wassers gelöst und $3,3 \mathrm{~g}$ d-Weinsäure (etwas mehr als für 1 Molekül berechnet - 3,19 $\mathrm{g}-$ ), in $10 \mathrm{ccm}$ Wasser gelöst, hinzugefügt. Nachdem die Lösung im Exsikkator über Schwefelsäure bis auf etwa $20 \mathrm{ccm}$ eingedunstet ist, scheiden sich grobkörnige Krystalle $\mathrm{ab}$, die abgesaugt und getrocknet $2,6 \mathrm{~g}$ wiegen. Sie werden aus kochendem Wasser bis zum konstanten Schmelzpunkt zweimal umkrystallisiert und zur Analyse im Vakuumexsikkator über Schwefelsäure getrocknet.

0,1461 g Substanz geben 0,2076 $\mathrm{g} \mathrm{CO}_{2}$ und 0,0647 $\mathrm{g} \mathrm{H}_{2} \mathrm{O}$.

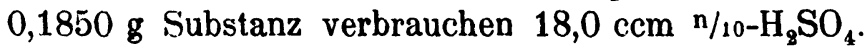

Berechnet für $\mathrm{C}_{6} \mathrm{H}_{9} \mathrm{~N}_{3} \mathrm{O}_{8} \cdot \mathrm{C}_{4} \mathrm{H}_{6} \mathrm{O}_{6}(305,16:)$ Gefunden:
C: $39,33 \%$
C: $38,75 \%$
$\mathrm{H}: \quad 4,95 \%$
$\mathrm{H}: \quad 4,91 \%$
$\mathrm{N}: 13,77 \%$
$\mathrm{N}: 13,63 \%$.

F.: Erweicht gegen $225^{\circ}$ (unkorr. $=229^{\circ} \mathrm{korr}$ ). Bei langsamem Erhitzen F. gegen 221-222 ${ }^{\circ}$. Zersetzt sich unter Aufschäumen gegen $230^{\circ}$ (unkorr. $=235^{\circ}$ korr.). Nach der Abspaltung der Weinsäure (s. unten) ergibt sich, daß das saure d-weinsaure d-Histidin auskrystallisiert ist.

Die Verbindung ist schwer löslich in kaltem Wasser, leicht in kochendem und verdünntem Alkohol; unlöslich in Äther, Aceton, Chloroform. Sie schmeckt säuerlich süß und läßt sich dadurch leicht von der l-Komponente unterscheiden.

Aus Wasser krystallisiert sie in länglichen, ungleichseitigen sechseckigen Tafeln von Wetzsteinform. Das spezifischeDrehungs- 
vermögen der weinsauren Histidinsalze nimmt mit fallender Konzentration anscheinend $z u$, ebenso wie bei den salzsauren Salzen.

Beim Ilistidinmonochlorhydrat beobachteten wir folgence Werte, berechnet auf die wasserfreie Substanz:
$\left.\mathrm{c}^{1}\right)=7,6048$
$[\alpha]_{\mathrm{D}}^{20^{\circ}}=+1,05^{\circ}\left( \pm 0,26^{\circ}\right)$
c $=7,6194$
$[\alpha]_{\mathrm{D}}^{20^{0}}=+1,18^{0}\left( \pm 0,26^{\circ}\right)$
c $=3,5235$
$[\alpha]_{\mathrm{D}}^{20^{\circ}}=+2,27^{\circ}\left( \pm 0,57^{\circ}\right)$
$\mathrm{c}=3,4971$
$[\alpha]_{D}^{200}=+4,00^{\circ}\left( \pm 0,57^{\circ}\right)$.

In einer früheren Arbeit ${ }^{2}$ ) fand der eine von uns $c=7,661$ $[\alpha]_{\mathrm{D}}^{20^{\circ}}=+1,70^{\circ}\left( \pm 0,26^{\circ}\right)$.

Für saures d-weinsaures d-Histidin erhielten wir folgende Zahlen :

I. $0,2610 \mathrm{~g}$ Substanz in $8,3108 \mathrm{~g}$ wässeriger Lösunई, $\mathrm{d}=1,0138$, drehten im $1 / 2 \mathrm{dm}-$ Rohr $+0,19^{\circ}\left( \pm 0,01^{\circ}\right)$.

II. $0,2278 \mathrm{~g}$ in $9,1998 \mathrm{~g}$ Lösung, $\mathrm{d}=1,0091, \alpha=+0,15^{\circ}$ $\left( \pm 0,01^{\circ}\right)$ im $1 / 2 \mathrm{dm}$-Rohr.

III. $0,2438 \mathrm{~g}$ in $11,0144 \mathrm{~g}$ lösung, $\mathrm{d}=1,0086$, drehte1 im $1 \mathrm{dm}-\mathrm{Rohr}+0,34^{\circ}\left( \pm 0,02^{\circ}\right)$.
I. $\mathrm{c}=3,1788$
$[\alpha]_{\mathrm{D}}^{20^{\circ}}=+11,95^{\circ}\left( \pm 0,73^{0}\right)$
II. $\mathrm{c}=2,4986$
$[\alpha]_{\mathrm{D}}^{20^{\circ}}=+12,01^{\circ}\left( \pm 0,80^{\circ}\right)$
III. $\mathrm{c}=2,2325$
$[\alpha]_{\mathrm{D}}^{200}=+15,23^{\circ}\left( \pm 0,90^{\circ}\right)$.

Die verarbeitete $d$-Weinsäure ergab für $c=2,9392$ $[\alpha]_{\mathrm{D}}^{20^{\circ}}=+17,01^{0}( \pm 0,68)$.

Die Mutterlauge des sauren d-weinsauren d-Histidins wurde auf dem Wasserbade bis zum Sirup eingeengt und dieser mit absolutem Alkohol verrieben. Es schieden sich bald weiße, feine Krystalle aus, die aus verdünntem Alkohol umkrystallisiert wurden und alle Eigenschaften des sauren d-weinsauren 1-Histidins zeigten.

1) $\mathrm{c}=$ Konzentration.

2) Emil Abderhalden und Hans Einbeck, a. a. 0. 
0,1256 g Substanz verbrauchen nach Kjeldahl 12,25 ccm $\mathrm{n} / 10-\mathrm{H}_{2} \mathrm{SO}_{4}$.

Berechnet für $\mathrm{C}_{6} \mathrm{H}_{9} \mathrm{~N}_{3} \mathrm{O}_{2} \cdot \mathrm{C}_{4} \mathrm{H}_{6} \mathrm{O}_{6}(305,16)$ : Gefunden:

$$
\mathrm{N}=13,77 \% \quad \mathrm{~N}=13,65 \% \text {. }
$$

F.: Erweicht gegen $175^{\circ}$ (unkorr.); zersetzt sich unter Aufschäumen gegen $180^{\circ}$ (unkorr.).

I. 0,4152 $\mathrm{g}$ Substanz in $9,7276 \mathrm{~g}$ wässeriger Lösung, $\mathrm{d}=1,0224$, drehen im $1 \mathrm{dm}-\operatorname{Rohr}+0,75^{\circ}\left( \pm 0,02^{\circ}\right)$.

II. $0,2656 \mathrm{~g}$ in $8,1844 \mathrm{~g}$ Lösung, $\mathrm{d}=1,0136$, drehen im $1 / 2 \mathrm{dm}-\mathrm{Rohr}+0,25^{\circ}\left( \pm 0,01^{\circ}\right)$.

III. $0,1068 \mathrm{~g}$ in $10,6010 \mathrm{~g}$ Lösung, $\mathrm{d}=1,001$, drehen im $1 \mathrm{dm}-$ Rohr $+0,16^{\circ}\left( \pm 0,01^{\circ}\right)$.

IV. Siehe oben.
I. $\mathrm{c}=5,4934$
$[\alpha]_{\mathrm{D}}^{20^{\circ}}=+13,65^{\circ}\left( \pm 0,36^{\circ}\right)$
II. $c=3,2705$
$[\alpha]_{\mathrm{D}}^{20^{\circ}}=+15,29^{\circ}\left( \pm 0,61^{\circ}\right)$
III. $\mathrm{c}=1,0075$
$[\alpha]_{\mathrm{D}}^{20^{\circ}}=+15,89^{\circ}\left( \pm 1,00^{\circ}\right)$
IV. $c=1,9104$
$[\alpha]_{\mathrm{D}}^{20^{\circ}}=+15,70^{\circ}\left( \pm 1,1^{\circ}\right)$.

Spaltung der sauren weinsauren Salze des dl-Histidins in d- und l-Histidin.

$5 \mathrm{~g}$ dl-Histidin werden in $50 \mathrm{ccm}$ Wasser und $5 \mathrm{~g} \mathrm{~d}-W$ Weinsäure gelöst. Im Exsikkator wird auf etwa $30 \mathrm{ccm}$ eingeengt. Es scheiden sich nach drei Tagen gut ausgebildete, grobe Krystalle ab, die abgesaugt und auf Tonplatten abgepreßt werden. Sie wiegen getrocknet $4,3 \mathrm{~g}$. Ihr Zersetzungspunkt liegt bei $230^{\circ}$ (unkorr.). Der Geschmack läßt erkennen, daß das saure d-weinsaure d-Histidin zuerst auskrystallisiert ist. Die weitere Verarbeitung der Krystallfraktion und der Mutterlauge ist dieselbe. Sie werden in etwa $50 \mathrm{ccm}$ verdünnter Salzsäure gelöst und eine Stunde lang am Rückflußkühler gekocht. Nach dem Erkalten wird mit Sodalösung stark alkalisch gemacht und mit einem Überschuß einer konzentrierten alkoholischen Sublimatlösung gefällt. Der weiße Niederschlag wird mit vielem destilliertem Wasser salzfrei dekantiert, auf dem Filter nochmals gründlich mit kaltem Wasser ausgewaschen, in heißem Wasser 
suspendiert und unter beständigem Umrühren mit Schwefelwasserstoff zersetzt. Vom ausgefallenen Quecksilbersulfid wird abfiltriert, das Filtrat durch Durchsaugen von Luft vom Schwefelwasserstoff befreit, und dann unter vermindertem Druck bis auf etwa $10 \mathrm{ccm}$ eingeengt. Jetzt fügt man die dem Chlorgehalt entsprechende Menge n-Lithiumhydroxydlösung hinzu und engt auf dem Wasserbade vorsichtig bis auf wenige Kubikzentimeter ein. Nach dem Erkalten wird das Histidin durch etwa $50 \mathrm{ccm}$ absoluten Alkohol ausgefällt. Zur Analyse werden beide Komponenten nochmals aus wenig Wasser umkrystallisiert.

I. Mutterlauge. l-Histidin.

F.: Zersetzung unter Aufschäumen bei $279^{\circ}$ (unkorr.).

0,1430 g Substanz verbrauchten nach Kjeldahl 27,55 ccm $\mathrm{n} / 10-\mathrm{H}_{2} \mathrm{SO}_{4}$.

Berechnet für $\mathrm{C}_{6} \mathrm{H}_{9} \mathrm{~N}_{3} \mathrm{O}_{2}$ :

$$
\mathrm{N}=27,10 \% \quad \mathrm{~N}=26,99 \% \text {. }
$$

Gefunden:

$0,1964 \mathrm{~g}$ Substanz in $9,5270 \mathrm{~g}$ wässeriger Lösung, $\mathrm{d}=1,009$, drehen im $1 / 2 \mathrm{dm}-$ Rohr $-0,40^{\circ}\left( \pm 0,01^{\circ}\right)$.

$$
[\alpha]_{\mathrm{D}}^{20^{\circ}}=-38,46^{\circ}\left( \pm 0,96^{0}\right) \text {. }
$$

Il. Krystallfraktion. d-Histidin.

F. : Zersetzung bei $278^{\circ}$ (unkorr.) unter Aufschäumen.

$0,1658 \mathrm{~g}$ Substanz verbrauchten $31,9 \mathrm{ccm} \mathrm{n} / 10-\mathrm{H}_{2} \mathrm{SO}_{4}$.

Berechnet: $\mathrm{N}=27,10 \% \quad$ Gefunden : $\mathrm{N}=26,97 \%$.

$0,1334 \mathrm{~g}$ Substanz in 7,5154 $\mathrm{g}$ wässeriger Lösung drehen im $1 / 2 \mathrm{dm}-\mathrm{Rohr}+0,31^{\circ}\left( \pm 0,02^{\circ}\right) . \mathrm{d}=1,002$.

$$
[\alpha]_{\mathrm{D}}^{20^{\circ}}:+34,88^{\circ}\left( \pm 2,24^{\circ}\right) \text {. }
$$

$0,2064 \mathrm{~g}$ Substanz in 8,0672 $\mathrm{g}$ Lösung, $d=1,0065$, drehen im $1 / 2 d-R o h r+0,50^{\circ}\left( \pm 0,01^{\circ}\right)$.

$$
[\alpha]_{\mathrm{D}}^{20^{\circ}}:+38,83^{\circ}\left( \pm 1,07^{\circ}\right) \text {. }
$$

Das so gewonnene d-Histidin stimmt in seinen physikalischen und chemischen Eigenschaften vollständig mit dem auf S. 447 beschriebenen, auf biologischem Wege erhaltenen überein.

Die Ausbeuten an reinem $1-$ und d-Histidin betragen cia. $60 \%$ der Theorie. 
Nach der Drucklegung der vorliegenden Arbeit und bereits erfolgten 1. Korrektur wurden wir auf die schon 1911 erschienene Arbeit von Frank Lee Pyman ${ }^{1}$ ) aufmerksam gemacht. Diesem Autor ist es gelungen, das dl-Histidin synthetisch darzustellen. Pyman hat den Racemkörper mit d-Weinsäure in seine beiden optisch-aktiven Komponenten zerlegt. Der Teil unserer Mitteilung, der von der Spaltung von dl-Histidin mittels $\mathrm{d}$-Weinsäure handelt, ist somit nur eine Bestätigung und zum Teil eine Ergänzung der von Pyman erhobenen Befunde.

1) Frank Lee Pyman, Die Synthese des Histidins. Journal of Chem. Soc., London, Bd. 99, S. 1386, 1911. 